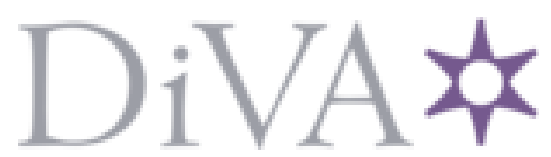

http://www.diva-portal.org

\title{
Postprint
}

This is the accepted version of a paper presented at CSCW'16 Computer Supported Cooperative Work and Social Computing San Francisco, CA, USA — February 27 - March 02, 2016.

Citation for the original published paper:

Handberg, L., Gullström, C., Kort, J., Nyström, J. (2016)

Spatial and Social Connectedness in Web-based Work Collaboration.

In: Darren Gergle, Meredith, Ringel Morris (ed.), Proceedings of the 19th ACM Conference on

Computer Supported Cooperative Work and Social Computing Companion: Interactive Demo (pp.

45-48). New Yor NY: ACM Digital Library

$\mathrm{ACM}$

http://dx.doi.org/10.1145/2818052.2874321

N.B. When citing this work, cite the original published paper.

(C) Handberg, Gullström, Kort, Nyström $\mid$ ACM 2016. This is the author's version of the work. It is posted here for your personal use. Not for redistribution. The definitive Version of Record was published in Proceedings of the 19th ACM Conference on Computer Supported Cooperative Work and Social Computing Companion, http://dx.doi.org/10.1145/2818052.2874321.

Permanent link to this version:

http://urn.kb.se/resolve?urn=urn:nbn:se:kth:diva- 185587 
Images below:

Screenshots from the SharedSpaces video with additional explanatory images

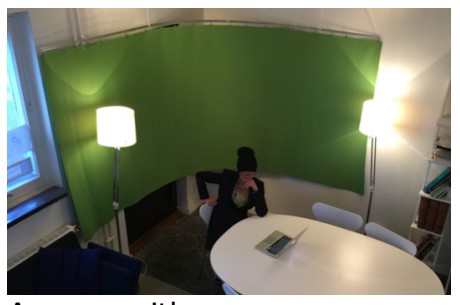

A room with a green screen and participant 1.

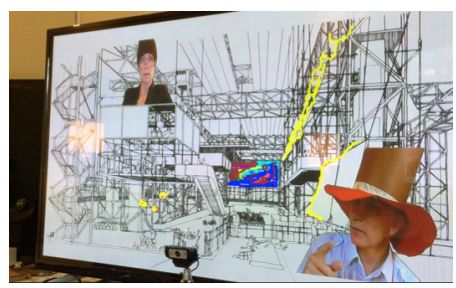

Shot of screen with two participants in SharedSpaces.

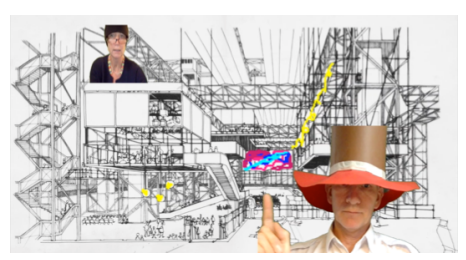

Screenshot from the same screen.

\section{Spatial and Social Connectedness in Web-based Work Collaboration}

\author{
Leif Handberg \\ Charlie Gullström \\ KTH \\ Stockholm \\ leifh@kth.se \\ charlieg@kth.se \\ Joke Kort \\ TNO \\ Groningen \\ joke.kort@tno.nl \\ Jimmy Nyström \\ LTU \\ Luleå \\ jimmy.nystrom@ltu.se
}

Paste the appropriate copyright/license statement here.

- License: The author(s) retain copyright, but ACM receives an exclusive publication license.

Each submission will be assigned a unique DOI string to be included here.

\begin{abstract}
The work presented here seeks an integration of spatial and social features supporting shared activities, and engages users in multiple locations to manipulate realtime video-streams. Standard and easily available equipment is used together with the communication standard WebRTC. It adds a spatial quality of experience by representing the users anywhere on the screen, with easily changed diverse backdrops, inviting users to co-design a shared mediated space. User studies show that a seamless integration of space, social dynamics and shared activity benefits the experience of presence, naturalness, immersion/ engagement and social connectedness. The results inform a discussion about spatial and social

connectedness, stressing the importance of design to integrate architectural/spatial features and support complex social dynamics in mediated interaction.
\end{abstract}

\section{Author Keywords}

Naturalness; immersion; presence; design; spatial connectedness; social connectedness, WebRTC; media, mediated space; audio/video communication.

\section{ACM Classification Keywords}

H.5.m. Information interfaces and presentation (e.g., $\mathrm{HCI})$ : Miscellaneous. 


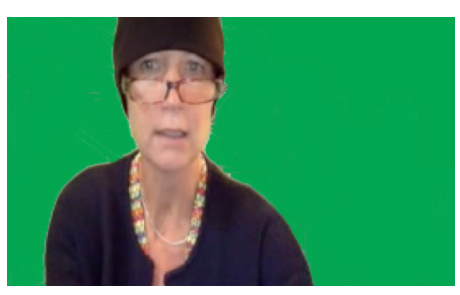

Camera view of participant 1 without green screen background removal.

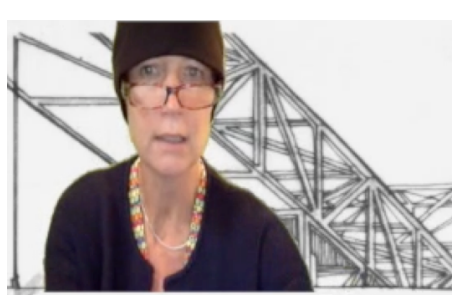

Camera view of participant with green screen background removal.

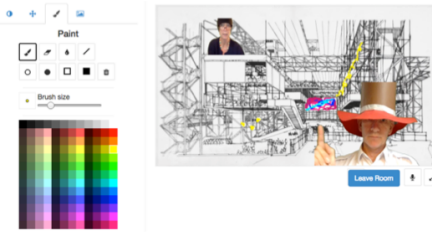

Drawing possible with the drawing tool.

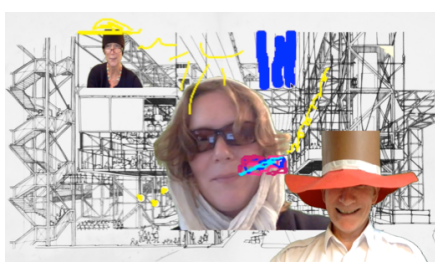

Third person enters.

\section{Introduction}

In the last decade there has been numerous attempts to reach beyond the "talking heads" and the 16:9 paradigm [12]. The most elaborate solutions have integrated heavy hardware in spaces where users benefit from how activity and social space are brought together through design, yet in very costly interiors

[3]. On the other hand, new standards for web-based communication (WebRTC, HTML5) facilitate a

representation of participants and activity space, simply via a web interface, with the help of a webcam and computer. While focus in further development of many web applications for mediated interaction is on technical maturation (e.g. automatic bandwidth reduction, switching microphone on/off through sound detection algorithms, adding additional tools for cooperative tasks, etc.), we hypothesize that multiparty interaction, and in particular naturalness [11], and immersion/engagement, will benefit from spatial, social and shared activity features in one seamless (virtual) space. Our ongoing design research specifically addresses the fusion of

- spatial connectedness (a term describing how well users perceive that real space is integrated with virtual space) and

- social connectedness (how well users perceive social dynamics/cues and their relationship with others in mediated space during interaction).

Our aim in the larger context is to create a seamless mediated space for social contact and shared activity by integrating live and/or stored media streams combined with software applications (e.g. physical sensors and actuators) that empower users to actively control features in local (as well as remote) and virtual spaces. Our aim for this work is to explore how well our design prototype SharedSpaces serves the seamless merging of spaces, social interaction and shared activities in a virtual space, and consequently enhances the experience of (social) presence, naturalness, immersion/engagement and social connectedness.

\section{Our work in context}

Over twenty-five years have passed since Bill Buxton and his colleagues [1] used architectural spaces as a reference for the Hydra studies in which they showed the importance of spatial cues and design as a prerequisite for presence in shared 'person spaces' and remote collaboration. They challenged media, as a new field, to achieve what architecture as a mature discipline already does: support physical proximity by activity-based design. Activities are central to the experience of social connectedness, of engaged relationships with others as well, addressed by Goffman as Interaction rituals (recurring activities that are the building blocks of our social habits, and can have varying frequencies of occurrence) $[5,6]$ and by Clark as shared activities and grounding (social dynamics and cues needed in interaction) [4]. Today, the field of presence is arguably mature - nevertheless still lacks in adequate terminology to also discuss how spatial connectedness and the experience of proximity and activity benefit and support the social connectedness. Spatial connectedness indicates that architectura design is as important in mediated environments as in real spaces for human interaction. In architecture, spatial features such as proximity, visibility and layout may stimulate interaction and collaboration [8]. Architectural design equally creates spatial dividers, walls and barriers by e.g. geographically separating offices or domestic environments, seeking to harbor privacy, security, but also (social) exclusion and loneliness $[13,14,2]$. Equally, in shared mediated 


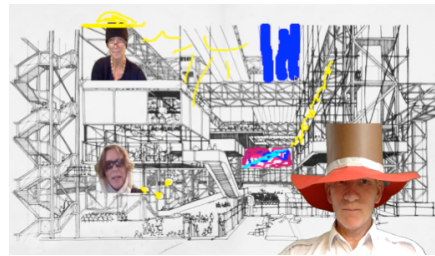

Third person scaled and moved with video control tool.

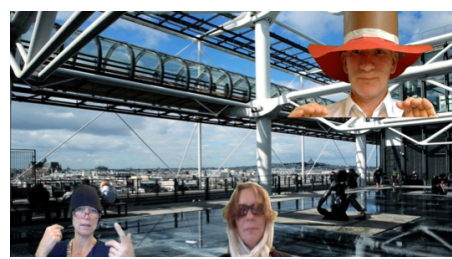

Change of background.

Backgrounds can be chosen from a predifined set of images, or be uploaded from any of the participants computers. The background can also be a live or recorded video feed. The background can be altered to support collaboration and

communication context and to help a conversation move between formal and informal stages.

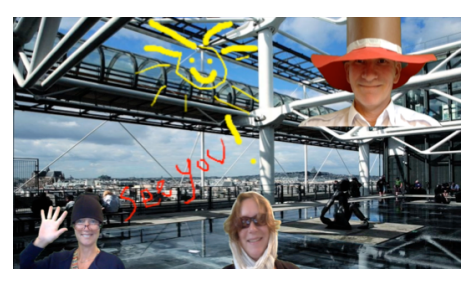

spaces, the individual experience of presence is negotiated as part of a process to establish trust and confirmation from the remote party/parties $[7,9,10]$. An ongoing construction of a sense of place is informed by a series of spatial and social cues, i.e. 'reality tests' carried out by participants, who seek fast, consistent and reliable confirmation that the interaction can be trusted - in which case they can temporarily agree to suspend their judgment in favor of a 'willing suspension of disbelief'. In a broader context, social connectedness is a layered construct that describes how people relate and connect with one another in society and social groups [14]. The linking factor between spatial and social connectedness is the ambition to design mediated spaces that support and enhance trustbuilding processes and secure this common ground and interaction rituals.

\section{Aim of the work}

Our aim for this work is to explore how well our design prototype SharedSpaces serves the seamless merging of spaces, social interaction and shared activities in a virtual space, enhancing the experience of (social) presence, naturalness, immersion and engagement and social connectedness.

\section{SharedSpaces design}

SharedSpaces adds to the quality of experience of spatial and social connectedness by representing multiple users positioned anywhere on the screen, with the possibility to customize their size and the backdrop. This engages users in manipulating their real-time video-streams, creatively co-designing a shared mediated space (media space) that fits their needs. It supports social dynamics by allowing users to change position and resize video streams and enhances grounding and social cues by merging video-streams and space, representing users as if they were "side by side".

Video-streams are merged in the virtual space in this WebRTC application with a green-screen component for background removal. New backdrops can easily be uploaded as images or live-feeds. Since the manipulation of video-streams is so easy, a user could also host others in his/her real space by simply removing the green background in his/her physical space or adding an additional video-stream of his/her space and maximizing this video-stream using it as a backdrop. Others will be merged into this backdrop. Furthermore, users can draw and paint on the screen/space as a whole, creating numerous possibilities for co-creation and strengthening social exchange.

All that users need to use this web application is a computer with webcam, an Internet connection, Google Chrome (or another browser supporting WebRTC), and a green background. No additional software needs to be installed.

\section{Conclusion - relevance to the CSCW audience}

In sum, the most important prerequisites for spatial and social connectedness are: a sense of shared space is established (presence); and to the extent that necessary cues needed for social interaction, grounding, and common understanding are secured (social presence); as well as a sense of naturalness in relation to the environment as a whole and to the individual self (naturalness); and an integration of activities (interaction rituals) that express social 
dynamics and create involvement (immersion \& engagement).

Our future design research will focus on enhancing our current prototype based on this work and other experiments and to further explore the relation between spatial and social connectedness in the context of a wider range of shared activities and in different domains (work, domestic and public environment).

\section{Acknowledgements}

This work was performed within the EU FP7 COMPEIT project (www.compeit.eu). The COMPEIT project explores the future of connected media and creates a web-based system for highly interactive, personalized, shared media experiences. The project is funded by the European Union.

\section{References}

1. Bill W. Buxton. 1992. Telepresence: integrating shared task and person spaces. In the Proceedings of Graphics Interface '92, 123-129.

2. John T. Cacioppo and William Patrick. Loneliness: Human Nature and the Need for Social Connection. 2009. W. W. Norton \& Company.

3. Cisco Immersive Telepresence. Rerieved September 24, 2015 from http://www.ivci.com/products/cisco/ciscoimmersive-telepresence/

4. Herbert H. Clark. 1996. Using language. Cambridge University Press.

5. Erving Goffman. 1961. The presentation of self in everyday life. New York, Doubleday.
6. Erving Goffman. 1967. Interaction ritual: Essays on face-to-face behaviour. Garden City, NY,

Doubleday.

7. Gullström, C, 2010. Presence design: Mediated spaces extending architecture. Doctoral dissertation. Stockholm: KTH Royal Institute of Techology.

8. Hillier, B. 1996. Space is the Machine: A Configurational Theory of Architecture (Cambridge: Cambridge University Press)

9. IJsselsteijn, W.A. (2004). Presence in depth. Doctoral dissertation. Eindhoven: Eindhoven University of ITechnology

10. Jsselsteijn, W.A., \& Riva, G. (2003). Being there: The experience of presence in mediated environments', In: G. Riva, F. Davide, \& W.A. IJsselsteijn (Eds.) Being there: Concepts, effects and measurement of user presence in synthetic environments. Amsterdam: IOS Press

11. Ned Kock. 2002. Evolution and Naturalness: A look at E-communication through a Darwinian Theoretical Lens. In Proceedings of the TwentyThird International Conference on Information Systems (ICIS'02).

12. Joke Kort, Charlie Gullstrom, Harold C. Nefs. 2013. Beyond talking heads - Towards sharing life. In the Proceedings of the SIGCHI Conference on Human Factors in Computing SYstems ( $\left.\mathrm{CHI}^{\prime} 13\right)$ workshop The Future of Personal Video Communication: Moving Beyond Talking Heads to Shared Experiences.

13. Marco C. Rozendaal and Nienke Hollenberg. 2013. Pediatric Oncology Sketched Out. TU Delft, Delft.

14. Sabine Wildevuur, Dick van Dijk, Anne Äyväri, Mie Bjerre, Thomas Hammer-Jakobsen, and Jesper Lund. 2013. Connect - Design for an Emphatic Society. BIS Publishers. 